\title{
Hemodynamic Changes During Neuromuscular Electrical Stimulation and Mobility Therapy for an Advanced Heart Failure Patient with Impella 5.0 Device A Case Report
}

\author{
Kohei Nozaki, ${ }^{1}$ PT, Nobuaki Hamazaki, ${ }^{1}$ PT, Yuki Ikeda, ${ }^{2}$ MD, Manami Nihei, ${ }^{1}$ PT, \\ Shuken Kobayashi, ${ }^{1}$ PT, Kentaro Kamiya, ${ }^{3}$ PT, Emi Maekawa, ${ }^{2}$ MD, Atsuhiko Matsunaga, ${ }^{3}$ PT, \\ Minako Yamaoka-Tojo, ${ }^{3} \mathrm{MD}$ and Junya Ako, ${ }^{2} \mathrm{MD}$
}

\begin{abstract}
Summary
The Impella 5.0 is a catheter-mounted left ventricular assist device that is inserted through the patient's subclavian artery. This device allows patient mobilization. Early mobility improves outcomes, including physical function and exercise tolerance, in critically ill patients and those with heart failure (HF). However, there have been no studies regarding the safety of early mobilization during the period of Impella 5.0 insertion based on hemodynamic assessment.

A 39-year-old man with idiopathic dilated cardiomyopathy and cardiogenic shock was transferred to our hospital for Impella 5.0 insertion. We started neuromuscular electrical stimulation (NMES) and mobilization eight days after Impella 5.0 insertion. The safety of NMES and mobilization was assessed based on mean blood pressure, heart rate (HR), and mean pulmonary artery pressure measurements as hemodynamic indicators. Muscle strength was also assessed using the Medical Research Council (MRC) scale. Throughout the interventions, only the HR increased slightly during mobilization, and there were no hemodynamic abnormalities. Also, the MRC scale score improved as mobilization progressed. The results presented here suggest that NMES and mobilization are safe and feasible in patients with Impella 5.0 insertion, and therefore should be widely adopted.
\end{abstract}

Key words: Cardiogenic shock, Safety, Feasibility, Early mobilization, Intensive care

(Int Heart J 2021; 62: 695-699)

$\mathrm{T}$ he Impella 5.0 catheter-mounted left ventricular assist device is increasingly being used in patients with cardiogenic shock. One major advantage of Impella 5.0 insertion through the axillary artery or subclavian artery is that patients can undergo mobilization while using the device, ${ }^{1)}$ and such early mobilization has beneficial effects on outcomes mortality. ${ }^{2)}$ Neuromuscular electrical stimulation (NMES) may be a method for both endurance and strength training in patients in the acute phase of advanced heart failure (HF). ${ }^{3)}$ However, there have been no reports regarding the safety of mobilization and NMES in patients using this device.

\section{Case Report}

A 39-year-old man with idiopathic dilated cardiomyopathy was admitted to our hospital due to acute decompensated HF and cardiogenic shock. His HF depended on catecholamine infusion, and we initially considered him for a heart transplant. However, based on his high degree of obesity (body mass index: 34.5), we found he was not eligible for transplantation. Therefore, we decided to use an Impella device to introduce beta-blockers and reverse remodeling of the myocardium. He was transferred to our hospital 85 days after admission to the previous hospital.

Findings on admission to our hospital with a high dose of catecholamine (i.e., milrinone, $0.3 \gamma$; dobutamine, $3.5 \gamma$; and dopamine, $3.0 \gamma$ ) were as follows: Glasgow Coma Scale, 14; left ventricular ejection fraction (LVEF), 15\%; brain natriuretic peptide (BNP) level, $1016 \mathrm{pg} / \mathrm{mL}$; New York Heart Association (NYHA) Functional Classification, IV; blood pressure (BP), 121/53 mmHg; heart rate (HR), 113 bpm (sinus rhythm); mean pulmonary pressure, $31 \mathrm{mmHg}$; cardiac index, (Fick method), 1.68; Forrester's hemodynamic subset, IV; Barthel index, 40 points. Since

From the 'Department of Rehabilitation, Kitasato University Hospital, Sagamihara, Japan, ${ }^{2}$ Department of Cardiovascular Medicine, Kitasato University School of Medicine, Sagamihara, Japan and ${ }^{3}$ Department of Rehabilitation, School of Allied Health Sciences, Kitasato University, Sagamihara, Japan.

This study was supported by a Grant-in-Aid [JSPS KAKENHI Grant Number JP 19K19884] from the Japan Society for the Promotion of Science.

Address for correspondence: Kohei Nozaki, PT, Department of Rehabilitation, Kitasato University Hospital, 1-15-1 Kitasato, Minami-ku, Sagamihara, Kanagawa, 252-0375 Japan. E-mail: 0818.n.kohei@gmail.com

Received for publication September 14, 2020. Revised and accepted November 9, 2020.

Released in advance online on J-STAGE May 15, 2021.

doi: 10.1536/ihj.20-632

All rights reserved by the International Heart Journal Association. 


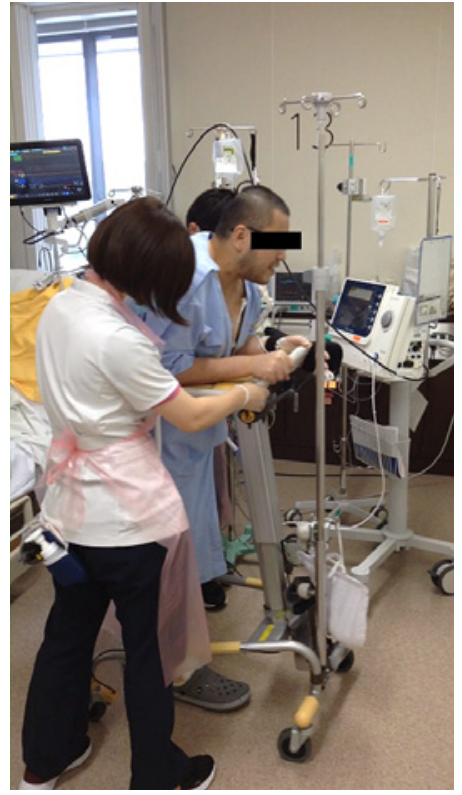

Figure 1. Mobility therapy (walking) in the patient during the period of Impella 5.0 insertion.

tapering catecholamines could easily lead to a systolic BP below $100 \mathrm{mmHg}$ and become shock vital signs, three days after admission, he was intubated, and we inserted the Impella 5.0 via the left subclavian artery.

We monitored mean BP, HR, and mean pulmonary artery pressure (PAP) via a radial arterial catheter, 12-lead electrocardiography, and right heart catheter during NMES and mobilization. We compared these indices before, during, and after NMES and mobilization with an analysis of variance (ANOVA). Also, we evaluated position waveforms and motor waveforms of Impella 5.0. Furthermore, we assessed physical function based on the Medical Research Council (MRC) scale score.

NMES was applied to the muscle groups of both legs, including the quadriceps femoris, hamstrings, tibialis anterior, and triceps surae muscles, using belt electrode skeletal muscle electrical stimulation (Auto TENS Pro; Homer Ion Co., Ltd., Tokyo, Japan). ${ }^{4)}$ Six silicon-rubber electrode bands were wrapped around the proximal and distal parts of the thigh and distal parts of the lower leg and held in place with hook-and-loop fastener straps (Homer Ion Co.). We set the anode at the thigh's distal part, with the cathode set at the proximal part of the lower leg's thigh and distal part. The stimulator current waveform was designed to produce co-contractions in the lower extremities' muscle groups at a frequency of $20 \mathrm{~Hz}$ with a pulse width of $250 \mu \mathrm{s}$ and a duty cycle involving stimulation for 5 seconds with a 2 -second pause. As stimulation cycles for the bilateral legs were synchronized, both legs were stimulated simultaneously. We set the output intensity to the maximum tolerable value without discomfort or pain to induce visible muscle contractions. NMES was performed for 30-40 minutes per day. The mobilization program was defined according to the statement of the Japanese Circulation Society as sitting over the edge of the bed, chair sitting, standing, and walking with a physical therapist (Figure 1). ${ }^{5}$

Figure 2 shows the clinical course in this case. All catecholamines could be withdrawn on day 9 after Impella 5.0 insertion. We introduced a beta-blocker two days after Impella 5.0 insertion and gradually increased the dose. NMES and mobilization (sitting over the edge of the bed and standing) were started eight days after Impella 5.0 insertion as the patient had a persistent high drug-induced fever for seven days. Walking was started 16 days after insertion. At least one of the two treatments was performed daily until the Impella 5.0 device was withdrawn on day 20 after insertion. The MRC scale score improved from 24 to 56 as the level of mobilization increased.

The total times of NMES and mobility sessions were 9 and 10 times, respectively.

During NMES sessions, there were no significant mean BP, HR, or mean PAP changes over the whole treatment period (Figure 3).

Figure 4 shows the mean BP, HR, and mean PAP changes before, during, and after the initial sittingstanding and walking sessions for hemodynamic assessment by mobilization. Mean BP and mean PAP showed no changes with either intervention, but a slight but significant increase was observed in HR with both interventions $(P=0.034,0.010$, respectively). Also, there were no changes in position or motor waveform during NMES and mobilization, and no other adverse events occurred.

The patient was discharged 57 days after transfer to our hospital. The Table presents data regarding cardiac function, physical function, and exercise tolerance at discharge. His HF was compensated, and his cardiac function improved at discharge. Although his muscle strength and exercise tolerance decreased regarding physical performance, the patient could manage daily living activities (ADL) independently.

\section{Discussion}

This is the first case report regarding NMES safety and mobility therapy according to hemodynamic changes using right heart catheterization in a patient with an Impella 5.0 device. Consistent with the present study's findings, Kamiya, et al. reported there were no changes in hemodynamics in patients with an implanted left ventricular assist device undergoing NMES. ${ }^{6}$ The increase in HR during mobilization appeared to be within the normal range of response to mobilization. Importantly, mobilization did not cause excessive fluctuations in mean BP or PAP. These interventions did not affect the hemodynamics in the present case, suggesting that NMES and mobilization were safe and feasible in patients with an Impella 5.0 device's insertion.

Impella is usually used to stabilize patients with cardiogenic shock or a bridge to implantation of a left ventricular assist device. These patients are at high risk of long-term inactivity, resulting in reductions in physical function, exercise tolerance, and quality of life (QoL). However, there is still no expert consensus on safety criteria for critically ill patients' mobilization regarding the Impella device. ${ }^{7)}$ NMES and mobilization are necessary to 


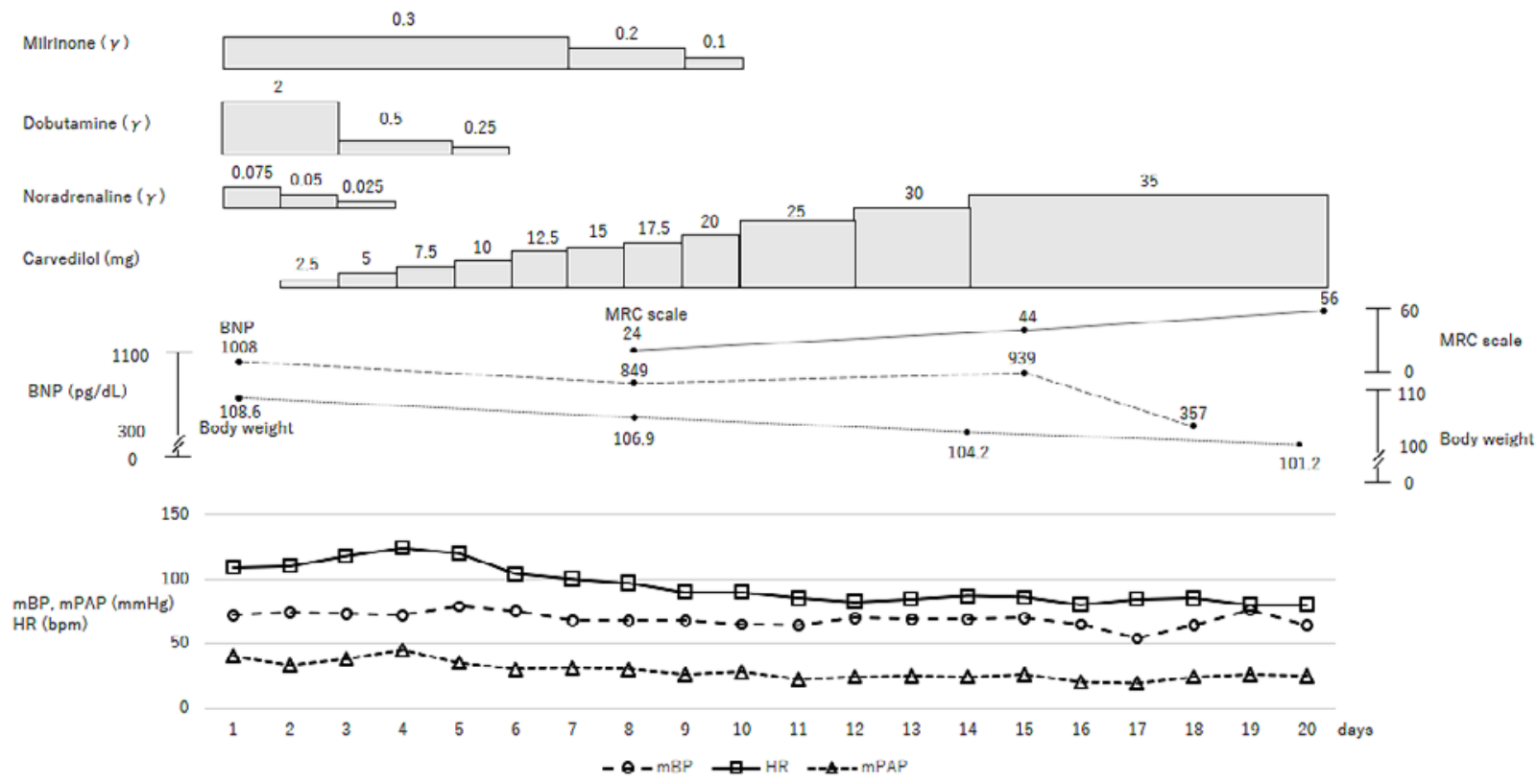

Days after Impella 5.0 insertion

Mobilizatio

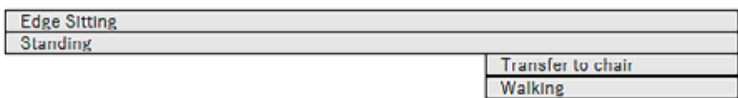

Figure 2. Clinical course. BNP indicates brain natriuretic peptide; HR, heart rate; mBP, mean blood pressure; mPAP, mean pulmonary artery pressure; and MRC, Medical Research Council.

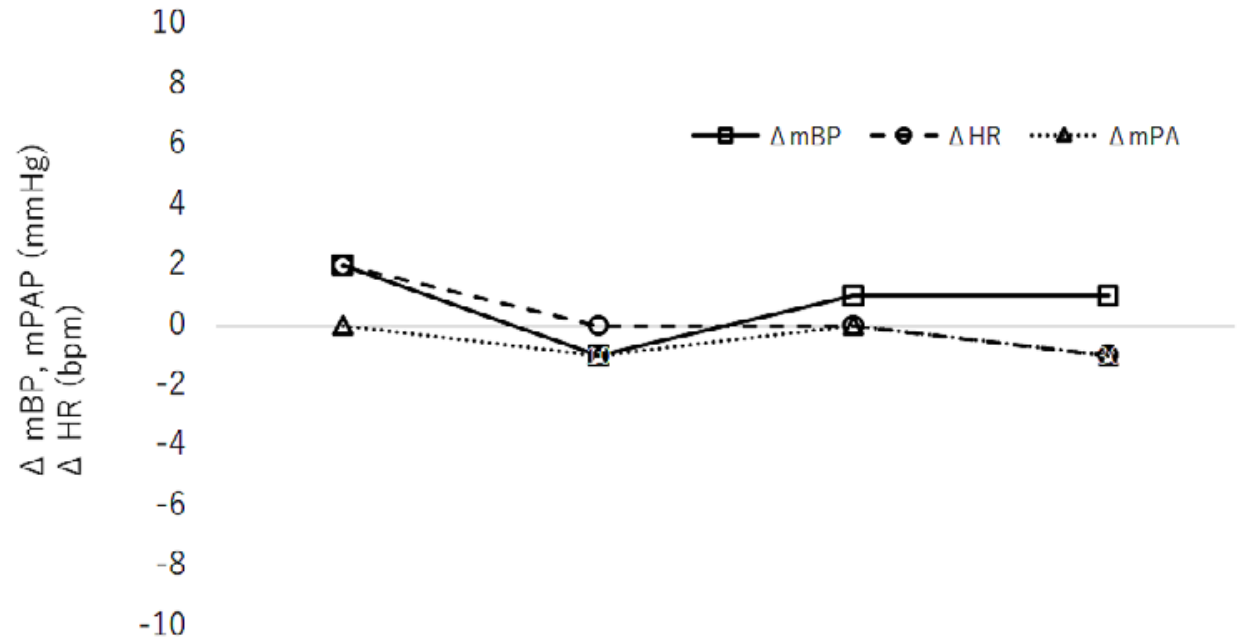
Baseline
$10 \mathrm{~min}$
$20 \min$
$30 \mathrm{~min}$

Figure 3. Hemodynamic parameters during neuromuscular electrical stimulation sessions. Data are presented as average for all sessions. HR indicates heart rate; $\mathrm{mBP}$, mean blood pressure; and mPAP, mean pulmonary artery pressure.

prevent the deleterious effects of intensive care, and this report may facilitate the proactive implementation of these interventions.

NMES reportedly increased muscle strength and exercise tolerance without adverse events in patients with chronic HF. ${ }^{3,8)}$ Kondo, et al. recently reported that starting NMES the day after admission was safe and feasible in patients with acute $\mathrm{HF}^{9)}$ However, this is the first report of the safety of NMES in patients an inserted Impella device. Most previous reports regarding the effects of NMES have been interventions lasting for several months, but Forestieri, et al. reported improved exercise tolerance after a 5-day intervention following cardiac surgery. ${ }^{10)}$ Therefore, it may be beneficial to treat patients with Im- 
Sitting, standing
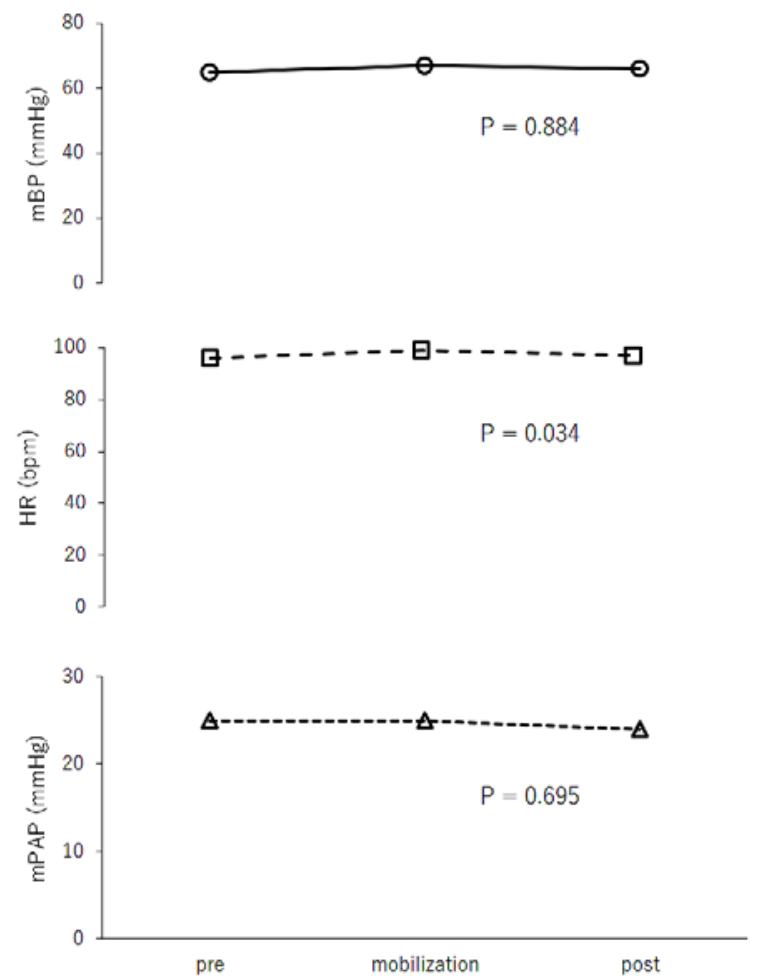

Walking
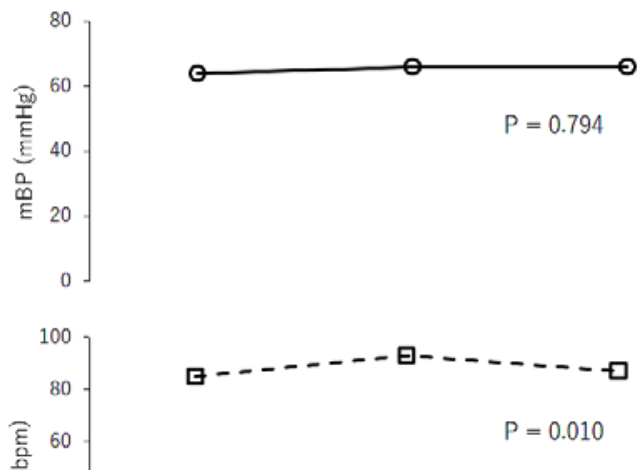

$P=0.010$

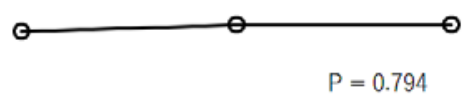

㕵 40

20.
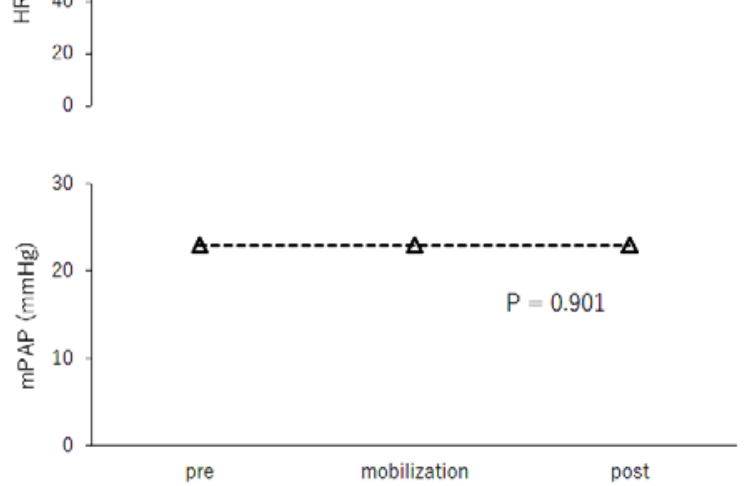

Figure 4. Changes in hemodynamic parameters before, during, and after initial sitting-standing and walking mobilization. HR indicates heart rate; $\mathrm{mBP}$, mean blood pressure; and $\mathrm{mPAP}$, mean pulmonary artery pressure.

Table. Cardiac Function, Physical Function, and Exercise Tolerance at the Time of Patient Transfer to Our Hospital and Discharge

\begin{tabular}{lcc}
\hline Variable & At transfer to our hospital & At discharge \\
\hline LVEF, $\%$ & 15 & 33 \\
Cardiac index & 1.68 & 2.31 \\
Forrester's subset & IV & $\mathrm{I}$ \\
BNP, pg/mL & 1016 & 147 \\
Hand grip, kg & Unable to measure & 26 \\
Gait speed, m/second & Unable to measure & 1.30 \\
Barthel index & 40 & 100 \\
6MWD, m & Unable to measure & 450 \\
Peak $\mathrm{VO}_{2}, \mathrm{~mL} / \mathrm{kg} /$ minute & Unable to measure & 15.1 \\
Peak $\mathrm{HR}_{2}$ beats/minute & Unable to measure & 143 \\
Peak $\mathrm{VO}_{2}, \mathrm{~mL} / \mathrm{beats} /$ hour & Unable to measure & 10.0 \\
VE/ $/ \mathrm{VCO}_{2}$ slope & Unable to measure & 31.7 \\
\hline
\end{tabular}

6MWD indicates six-minute walking distance; $\mathrm{BNP}$, brain natriuretic peptide; $\mathrm{HR}$, heart rate; LVEF, left ventricular ejection fraction; $\mathrm{VE} / \mathrm{VCO}_{2}$, minute ventilation/carbon dioxide production; and $\mathrm{Peak} \mathrm{VO}_{2}$, peak oxygen consumption rate.

pella 5.0 insertion for a short period with NMES. However, no data are available regarding the effects of NMES in patients with Impella 5.0 insertion, and further studies are required to address this issue.

A higher maximum level of mobility during Impella support was reportedly associated with an increased survival rate. ${ }^{2)}$ Mobilization in the intensive care unit (ICU) prevents prolonged hospitalization as well as ICUacquired weakness (ICU-AW), and to improve physical function, ADL and QOL of patients. ${ }^{11-13)}$ Therefore, care is required to avoid exacerbation of $\mathrm{HF}$ due to overload. Mobilization is important even in patients with an inserted Impella 5.0 device.

The muscle strength and exercise tolerance at discharge were poor in this patient, and he required continued outpatient cardiac rehabilitation. However, despite a prolonged ICU stay, the patient was independent in ADL. This may have been because NMES and mobilization dur- 
ing the period of Impella insertion prevented ICU-AW. The MRC scale score improved with mobilization, and muscle strength was good at Impella 5.0 withdrawal. As MRC scale score $<48$ is used to make a diagnosis of ICU-AW, this case did not present with ICU-AW at the time of Impella withdrawal. ${ }^{14)}$

In summary, NMES and mobilization for advanced HF patients with insertion of the Impella 5.0 cathetermounted left ventricular assist device via the subclavian artery might be safe and not induce hemodynamic disturbance. With appropriate risk management, these interventions may improve the physical function of patients without adverse events.

\section{Disclosure}

Conflicts of interest: The authors have no conflicts of interest to declare.

\section{References}

1. Tarabichi S, Ikegami H, Russo MJ, Lee LY, Lemaire A. The role of the axillary Impella 5.0 device on patients with acute cardiogenic shock. J Cardiothorac Surg 2020; 15: 218.

2. Esposito ML, Jablonski J, Kras A, Krasney S, Kapur NK. Maximum level of mobility with axillary deployment of the Impella 5.0 is associated with improved survival. Int $\mathbf{J}$ Artif Organs 2018; 41: 236-9

3. Sbruzzi G, Ribeiro RA, Schaan BD, et al. Functional electrical stimulation in the treatment of patients with chronic heart failure: a meta-analysis of randomized controlled trials. Eur J Cardiovasc Prev Rehabil 2010; 17: 254-60.

4. Tanaka S, Kamiya K, Matsue Y, et al. Effects of acute phase in- tensive electrical muscle stimulation in frail elderly patients with acute heart failure (ACTIVE-EMS): Rationale and protocol for a multicenter randomized controlled trial. Clin Cardiol 2017; 40: 1189-96.

5. Izawa H, Yoshida T, Ikegame T, et al. Standard cardiac rehabilitation program for heart failure. Circ J 2019; 83: 2394-8.

6. Kamiya K, Mezzani A, Masuda T, Matsunaga A, Izumi T, Giannuzzi P. Effects of electrical muscle stimulation in a left ventricular assist device patient. Int J Cardiol 2012; 160: e44-5.

7. Hodgson CL, Stiller K, Needham DM, et al. Expert consensus and recommendations on safety criteria for active mobilization of mechanically ventilated critically ill adults. Crit Care 2014; 18: 658 .

8. Smart NA, Dieberg G, Giallauria F. Functional electrical stimulation for chronic heart failure: a meta-analysis. Int J Cardiol 2013; 167: 80-6.

9. Kondo T, Yamada S, Tanimura D, et al. Neuromuscular electrical stimulation is feasible in patients with acute heart failure. ESC Heart Fail 2019; 6: 975-82.

10. Forestieri P, Bolzan DW, Santos VB, et al. Neuromuscular electrical stimulation improves exercise tolerance in patients with advanced heart failure on continuous intravenous inotropic support use-randomized controlled trial. Clin Rehabil 2018; 32: 6674.

11. Hermans G, De Jonghe B, Bruyninckx F, Van den Berghe G. Interventions for preventing critical illness polyneuropathy and critical illness myopathy. Cochrane Database Syst Rev 2014; CD006832.

12. Kayambu G, Boots R, Paratz J. Physical therapy for the critically ill in the ICU: a systematic review and meta-analysis. Crit Care Med 2013; 41: 1543-54.

13. Schweickert WD, Pohlman MC, Pohlman AS, et al. Early physical and occupational therapy in mechanically ventilated, critically ill patients: a randomised controlled trial. Lancet 2009; 373: $1874-82$.

14. Stevens RD, Marshall SA, Cornblath DR, et al. A framework for diagnosing and classifying intensive care unit-acquired weakness. Crit Care Med 2009; 37: S299-308. 